\title{
Edema and myocardial necrosis evaluated by cardiac magnetic resonance in acute myocarditis. Relationship with biomarkers and ventricular function
}

\author{
Joao S Marques, Ana G Almeida*, Raquel Magalhaes, Doroteia R Silva, Manuel G Varela, Susana Martins, \\ Dulce A Brito, Antonio N Diogo
}

From 2011 SCMR/Euro CMR Joint Scientific Sessions

Nice, France. 3-6 February 2011

\section{Introduction}

Acute myocarditis has a wide spectrum of clinical presentations. Knowledge of temporal and spatial evolution of inflammation and myocardial necrosis and its correlation with the systemic inflammatory markers may help to recognize patients at risk for progression to chronic cardiac dysfunction.

\section{Purpose}

Our objective was to evaluate if serum biomarkers and markers of myocardial necrosis can predict oedema and necrosis assessed by cardiac magnetic resonance (CMR).

\section{Methods}

We included 31 consecutive patients admitted with the diagnosis of acute myocarditis, confirmed by CMR in the presence of the following criteria: a) chest pain and/ or heart failure, b) elevated markers of myocardial necrosis, c) normal coronary angiography. Clinical and laboratorial evaluation (high sensitivity CRP, NTpro-BNP, troponin I, CK, CK-MB, serology and antibodies) was carried out. Patients underwent evaluation by CMR with determination of ejection fraction and wall motion score index $($ WMS $)=(\Sigma$ Score of each segment $/$ 17). CMR late gadolinium enhancement (LGE) was used to assess necrosis and a necrosis index (IN) was derived (LGE mass/total myocardial mass). Myocardial oedema was assessed visually from T2W sequence images. Oedema (OS) and necrosis (NS) scores were derived in a segmental basis $(1=$ presence, $2=$ absence in each segment; $\Sigma$ Score of each segment/17).

\section{Results}

All patients (26 [[Unsupported Character - \&\#9794;]], 5 [[Unsupported Character - \&\#9792;]], 26.3 \pm 12.5 years) showed an increase of at least one of the markers of necrosis (100\% with elevated troponin levels (16.1 $\pm 11.6 \mathrm{ug} / \mathrm{ml}))$. CRP was increased in $93 \%(6.2 \pm 4.4 \mathrm{mg} / \mathrm{dl})$, $84 \%$ had elevation of NTproBNP $(551.7 \pm 434.0 \mathrm{pg} / \mathrm{ml})$. The ejection fraction $(58.4 \pm 8 \%, 8 \%)$ was slightly decreased in 7 patients and WMS was increased in only $2(1.16 \pm 0.22)$. Oedema $(\mathrm{OS}=0.29 \pm 0.08)$ and necrosis (NS $=0.26 \pm 0.11)$ in all patients. The index of necrosis was $5.6 \% \pm 1.7 \%$. We found a significant correlation between: NTproBNP and WMS $(\mathrm{R}=0.791 \mathrm{p}=0.002)$, NTproBNP and IN $(\mathrm{R}=0.756 \mathrm{p}=0.02)$, and troponin and IN $(R=0.688 p=0.005)$, CK and NS $(R=0.580 \mathrm{p}=0.017)$.

\section{Conclusions}

In a patients with myocarditis, in spite of a preserved ejection fraction in the majority, the extent of oedema and necrosis evaluated by CMR correlated with the levels of serum biomarkers. CMR is a potential tool to define prognosis in these patients.

Published: 2 February 2011

doi:10.1186/1532-429X-13-S1-P321

Cite this article as: Marques et al.: Edema and myocardial necrosis evaluated by cardiac magnetic resonance in acute myocarditis. Relationship with biomarkers and ventricular function. Journal of Cardiovascular Magnetic Resonance 2011 13(Suppl 1):P321.

University Hospital Santa Maria, Lisbon, Portugal

(c) 2011 Marques et al; licensee BioMed Central Ltd. This is an open access article distributed under the terms of the Creative Commons 